\title{
Multiple-Symbol Detection Aided Differential Spatial Division Multiple Access
}

\author{
Li Wang and Lajos Hanzo, Fellow, IEEE \\ School of ECS, University of Southampton, SO17 1BJ, UK. \\ Tel: +44-23-8059 3125, Fax: +44-23-8059 4508 \\ Email: $\{1 w 05 r, 1 h\} @$ ecs.soton.ac.uk; http://www-mobile.ecs.soton.ac.uk
}

\begin{abstract}
This paper presents a multiple-symbol differential spatial division multiple access (MS-DSDMA) system conceived for lowcomplexity and high-bandwidth-efficiency applications operating in timevarying fading channels, where no channel estimation is required. A low-complexity adaptive multiple-symbol differential interference suppression (MS-DIS) scheme is proposed, which is based on the maximum signal-to-interference-plus-noise ratio (MSINR) criterion and facilitates the implementation of the powerful multiple-symbol differential sphere detection (MSDSD). Then, a practical three-stage turbo DIS receiver design framework is proposed for the MS-DSDMA system, which is constituted by concatenating the adaptive DIS filter bank, the MSDSD and the channel decoder. Both the EXtrinsic Information Transfer (EXIT) chart analysis and the Monte-Carlo-based simulation results show that the proposed three-stage turbo DIS scheme is capable of achieving a substantially enhanced performance in comparison to the conventional linear minimum mean-squared error (LMMSE) based adaptive receiver. Furthermore, for the sake of significantly reducing the iterative detection complexity, two complexity reduction techniques are devised, namely the a priori-LLR-threshold (ALT) and the adaptive-window-duration (AWD) aided MSDSD schemes.
\end{abstract}

\section{INTRODUCTION}

Owing to the scarcity of spectral resources, one of the main objectives in the design of future communication systems is the efficient exploitation of the available spectrum, in order to accommodate the ever-increasing traffic demands. The most promising solution to achieve this goal is based on the exploitation of the spatial dimension, by using spatial division multiple access (SDMA) [1], where the userspecific channel impulse responses (CIRs) are estimated and invoked for differentiating the parallel uplink streams transmitted by the different users. However, it was revealed in [2] that the multiple-input multiple-output (MIMO) system's performance is highly sensitive to the channel estimation errors, which may only be mitigated at the cost of an excessive computational complexity and/or high pilot overheads in many practical time-varying fading scenarios. Fortunately, in costand complexity-constrained applications there are options to circumvent the channel estimation, where the multiple-access interference (MAI) may be estimated and exploited by an adaptive receiver using a training sequence for the desired user. For example, the adaptive minimum mean square error (MMSE) scheme [3] using the least mean square (LMS) or the recusive least squares (RLS) algorithm and the more recently proposed maximum signal-to-interferenceplus-noise ratio (MSINR) based differential interference suppression (DIS) scheme [4]. For the former the interference suppression filter is adapted in order to minimize the MSE between the transmitted signal and the filter's output signal, while for the latter the filter coefficients are adjusted to maximize the SINR at its output and has been demonstrated in [4] to be able to mitigate the effects of carrier phase variations. Although they differ in their concept, the MSINR solution subsumes its MMSE-based counterpart as a special case [5].

1). Firstly, inspired by the block least-squares algorithm of [3] designed for standard MMSE adaptation, a new adaptive multiplesymbol DIS (MS-DIS) scheme is proposed based on our multiplesymbol differential SDMA (MS-DSDMA) system model for the sake of reducing the filter adaptation overheads and, even more importantly, for facilitating the implementation of the low-complexity yet

Acknowledgements: The finacial support of the EPSRC UK under the auspices of the UK-India centre of Excellence in Wireless Communications and that of the EU Optimix project is gratefully acknowledged. powerful multiple-symbol differential sphere detector (MSDSD) of [6]. 2). Secondly, as a benefit of employing the MSDSD, extra coding gains inherent in differential encoded systems may be gleaned by exploiting the correlation between the phase distortions experienced by the consecutively transmitted symbols. In order to further exploit the differential coding gains in the context of our adaptive MS-DIS scheme, a new channel-code-aided three-stage turbo DIS receiver is proposed, which allows a beneficial information exchange amongst the concatenated adaptive MS-DIS filter bank, the MSDSD and the channel decoder. 3). Thirdly, the new apriori-LLR-threshold (ALT) based MSDSD scheme is conceived, which is further aided by the proposed adaptive-window-duration (AWD) technique in order to achieve significant complexity reductions in the turbo DIS receiver.

\section{Multiple-Symbol DSDMA System Model}

We consider a discrete-time, complex baseband DSDMA system transmitting in the uplink over flat fading channels, which supports $U$ differential-modulation-based $N_{t}$-antenna-aided mobile stations (MS) while having $N_{r}$ receive antennas at the base station (BS). Due to pratical cost and size constraints, the employment of a single transmit antenna is assumed for each MS, i.e. $N_{t}=1$. Hence, a narrowband single-symbol system model may be constructed for the $n$th symbol duration as follows:

$$
\mathbf{Y}[n]=\sum_{u=1}^{U} \mathbf{S}_{u}[n] \mathbf{H}_{u}[n]+\mathbf{W}[n],
$$

where $\mathbf{Y}[n] \in \mathbb{C}^{N_{t} \times N_{r}}, \mathbf{S}_{u}[n] \in \mathbb{C}^{N_{t} \times N_{t}}$ and $\mathbf{W}[n] \in \mathbb{C}^{N_{t} \times N_{r}}$ denote the received signal and transmitted signal matrix as well as the AWGN matrix having a distribution of $\mathcal{C N}\left(0,2 \sigma_{w}^{2}\right)$, respectively. The $u$ th single-antenna-aided MS differentially encodes its information symbols $\mathbf{V}_{u}[n] \in \mathcal{M}_{c}=\left\{e^{j 2 \pi m / M} ; m=0,1, \cdots, M-1\right\}$, each of which contains $\left(\log _{2} M\right)$-bit information, as $\mathbf{S}_{u}[n]=\mathbf{V}_{u}[n] \mathbf{S}_{u}[n-$ 1]. Furthermore, the CIR matrix $\mathbf{H}_{u}[n]$ is a $\left(N_{t} \times N_{r}\right)$-dimensional i.i.d. zero-mean unit-variance complex Gaussian matrix, which is also referred to as the user-specific spatial signature that has to be estimated in conventional SDMA systems. However, the CIR matrix $\mathbf{H}_{u}[n]$ is not required at either the MS or the BS in the DSDMA system considered for sake of circumventing the potentially excessive-complexity and yet inaccurate channel estimation.

Now, based on (1) we can also construct the multiple-symbol DSDMA system model as:

$\underline{\mathbf{Y}}\left[k_{N}\right]=\sum_{u=1}^{U} \underline{\mathbf{Y}}_{u}\left[k_{N}\right]+\underline{\mathbf{W}}\left[k_{N}\right]=\sum_{u=1}^{U} \underline{\mathbf{S}}_{u}^{d}\left[k_{N}\right] \underline{\mathbf{H}}_{u}\left[k_{N}\right]+\underline{\mathbf{W}}\left[k_{N}\right]$,

where the $k$ th received signal block matrix $\underline{\mathbf{Y}}\left[k_{N}\right]$ contains $N_{\text {wind }}$ consecutively received signal matrices. Hence we have $\underline{\mathbf{Y}}\left[k_{N}\right]=$ $\left[\mathbf{Y}\left[\left(N_{\text {wind }}-1\right)(k-1)\right]^{T} \ldots \mathbf{Y}\left[\left(N_{\text {wind }}-1\right) k\right]^{T}\right]^{T}$, and both the channel's $k$ th block matrix $\underline{\mathbf{H}}_{u}\left[k_{N}\right]$ as well as the AWGN's $k$ th block matrix $\underline{\mathbf{W}}\left[k_{N}\right]$ are defined by vertically stacking the $N_{\text {wind }}$ matrices $\mathbf{H}_{u}[n]$ and $\mathbf{W}[n]\left(n=\left(N_{\text {wind }}-1\right)(k-1), \cdots,\left(N_{\text {wind }}-1\right) k\right)$, respectively. Moreover, the $k$ th diagonal block matrix of the transmitted signal $\underline{\mathbf{S}}_{u}^{d}\left[k_{N}\right]$ of the $u$ th MS is constructed as $\underline{\mathbf{S}}_{u}^{d}\left[k_{N}\right]=$ $\operatorname{diag}\left\{\mathbf{S}_{u}\left[\left(N_{\text {wind }}-1\right)(k-1)\right] \cdots \mathbf{S}_{u}\left[\left(N_{\text {wind }}-1\right) k\right]\right\}$, which corresponds to the length- $\left(N_{\text {wind }}-1\right)$ information symbol block matrix $\underline{\mathbf{V}}_{u}\left[k_{N}\right]=\left[\mathbf{V}_{u}\left[\left(N_{\text {wind }}-1\right)(k-1)+1\right]^{T} \ldots \mathbf{V}_{u}\left[\left(N_{\text {wind }}-1\right) k\right]^{T}\right]^{T}$. 


\section{Multiple-Symbol Differential Spatial Division Multiple ACCESS}

It is observed from both (1) and (2) that non-coherent differential detection techniques cannot be directly applied at the BS to recover the information pertaining to a specific MS without suppressing the interference imposed by all the other MSs. Therefore, we will use the designed MSINR approach for interference suppression in the DSDMA system as advocated in [4]. However, rather than computing the $u$ th MS's linear vector filter $\mathbf{f}_{u}[n]$ for each symbol duration $n$, we propose updating $\mathbf{f}_{u}\left[k_{N}\right]$ only once for $N_{\text {wind }}$ symbol durations based on the most recently received $N_{\text {wind }}$ signal matrices hosted by $\underline{\mathbf{Y}}\left[k_{N}\right]$ of (2). The resultant new multiple-symbol MSINR (MSMSINR) criterion reduces the filter-update overhead and additionally facilitates the implementation of a low-complexity yet powerful multiple-symbol differential sphere detector (MSDSD) in the following stage, hence achieving significant performance improvements.

\section{A. Adaptive Multiple-Symbol Differential Interference Suppression}

1) Multiple-Symbol MSINR Criterion: First of all, let us now derive the MS-MSINR criterion with the aid of the multiple-symbol system model of (2). In order to extract $N_{\text {wind }}$ differentially encoded symbols transmitted consecutively by the desired user, namely the $v$ th MS, from the $k$ th block of $N_{\text {wind }}$ successively received symbols hosted by $\mathbf{Y}\left[k_{N}\right]$ of (2), $\underline{\mathbf{Y}}\left[k_{N}\right]$ is passed through a linear vector filter $\mathbf{f}_{v}\left[k_{N}\right]$ of length $N_{r}$, yielding the filter output $\mathbf{y}_{v}\left[k_{N}\right]$ of length $N_{\text {wind }}$ as:

$$
\mathbf{y}_{v}\left[k_{N}\right]=\underline{\mathbf{Y}}\left[k_{N}\right] \mathbf{f}_{v}\left[k_{N}\right]
$$

We define the multiple-symbol-based SINR as the ratio between the sum power of the $N_{\text {wind }}$ desired filter output components of $\underline{\mathbf{Y}}_{v}\left[k_{N}\right] \mathbf{f}_{v}\left[k_{N}\right]$ and the sum power of the $N_{\text {wind }}$ interference-plusnoise components of $\left(\underline{\mathbf{Y}}\left[k_{N}\right]-\underline{\mathbf{Y}}_{v}\left[k_{N}\right]\right) \mathbf{f}_{v}\left[k_{N}\right]$. Our goal is to find the specific filter $\mathbf{f}_{v}\left[k_{N}\right]$ capable of maximizing the filter's output SINR, which may be mathematically expressed as:

$$
\begin{aligned}
\mathbf{f}_{v}\left[k_{N}\right] & =\max _{\mathbf{f}_{v}\left[k_{N}\right]} \frac{\mathbf{f}_{v}^{H}\left[k_{N}\right]\left(\mathbf{R}\left[k_{N}\right]-\mathbf{R}_{v}^{i}\left[k_{N}\right]\right) \mathbf{f}_{v}\left[k_{N}\right]}{\mathbf{f}_{v}^{H}\left[k_{N}\right] \mathbf{R}_{v}^{i}\left[k_{N}\right] \mathbf{f}_{v}\left[k_{N}\right]}, \\
& =\max _{\mathbf{f}_{v}\left[k_{N}\right]} \frac{\mathbf{f}_{v}^{H}\left[k_{N}\right] \mathbf{R}\left[k_{N}\right] \mathbf{f}_{v}\left[k_{N}\right]}{\mathbf{f}_{v}^{H}\left[k_{N}\right] \mathbf{R}_{v}^{i}\left[k_{N}\right] \mathbf{f}_{v}\left[k_{N}\right]}
\end{aligned}
$$

where $\mathbf{R}\left[k_{N}\right]$ is the correlation matrix of the multiple-symbol-based received signal $\underline{\mathbf{Y}}\left[k_{N}\right]$ of (2), which is defined as

$$
\mathbf{R}\left[k_{N}\right] \triangleq E\left\{\underline{\mathbf{Y}}^{H}\left[k_{N}\right] \underline{\mathbf{Y}}\left[k_{N}\right]\right\}=\sum_{u=1}^{U} \underline{\mathbf{H}}_{u}^{H}\left[k_{N}\right] \underline{\mathbf{H}}_{u}\left[k_{N}\right]
$$

and the multiple-symbol-based interference-plus-noise correlation matrix $\mathbf{R}_{v}^{i}\left[k_{N}\right]$ may be expressed as

$$
\begin{aligned}
\mathbf{R}_{v}^{i}\left[k_{N}\right] & \triangleq E\left\{\left(\underline{\mathbf{Y}}\left[k_{N}\right]-\underline{\mathbf{Y}}_{v}\left[k_{N}\right]\right)^{H}\left(\underline{\mathbf{Y}}\left[k_{N}\right]-\underline{\mathbf{Y}}_{v}\left[k_{N}\right]\right)\right\}, \\
& =\sum_{u=1 ; u \neq v}^{U} \underline{\mathbf{H}}_{u}^{H}\left[k_{N}\right] \underline{\mathbf{H}}_{u}\left[k_{N}\right]+2 \sigma_{w}^{2} \mathbf{I}_{N_{r}} .
\end{aligned}
$$

Following a similar derivation to that in [5], the maximization problem of (4) leads to the generalized eigenvalue problem via the method of Lagrange multipliers, which may be written as:

$$
\mathbf{R}\left[k_{N}\right] \mathbf{f}_{v}\left[k_{N}\right]=\lambda \mathbf{R}_{v}^{i}\left[k_{N}\right] \mathbf{f}_{v}\left[k_{N}\right] .
$$

2) MS-MSINR-Based Differential Interference Suppression: It is observed from (5) and (7) that in coherent-detection-aided SDMA systems, channel estimation has to be carried out to acquire each MS's spatial signature $\underline{\mathbf{H}}_{u}\left[k_{N}\right]$ for the sake of determining the coefficients of the MS-MSINR filter $\mathbf{f}_{v}\left[k_{N}\right]$ by solving the generalized eigenvalue problem of (8) using the singular-value decomposition (SVD) [7].
Fortunately, despite dispensing with the channel estimation in the DSDMA system, the interference-plus-noise correlation matrix $\mathbf{R}_{v}^{i}\left[k_{N}\right]$ of (7) may be calculated by taking advantage of the differentially encoded transmission as follows:

$$
\mathbf{R}_{v}^{i}\left[k_{N}\right]=\mathcal{E}\left\{\underline{\mathbf{E}}_{v}^{H}\left[k_{N}\right] \underline{\mathbf{E}}_{v}\left[k_{N}\right]\right\}
$$

in which the multiple-symbol-based interference-plus-noise signal matrix $\underline{\mathbf{E}}_{v}\left[k_{N}\right]$ is defined as:

$$
\underline{\mathbf{E}}_{v}\left[k_{N}\right]=\sqrt{\frac{1}{2}}\left(\underline{\mathbf{Y}}\left[k_{N}\right]-\underline{\tilde{\mathbf{V}}}_{v}\left[k_{N}\right] \cdot \underline{\mathbf{Y}}\left[k_{N}^{-1}\right]\right),
$$

where ' 9 ' denotes the submatrix-wise multiplication of two matrices and the block index $k_{N}^{-n}$ represents the $k$ th block shifted backwards by $n$ symbol durations. Moreover, $\underline{\mathbf{V}}_{v}\left[k_{N}\right]=\left[\mathbf{V}_{v}\left[\left(N_{\text {wind }}-1\right)(k-\right.\right.$ 1) $\left.]^{T} \underline{\mathbf{V}}_{v}\left[k_{N}\right]^{T}\right]^{T}$ is the transmitted information symbol block matrix of the $v$ th MS, which becomes known to the receiver during the training session or may be estimated by using the previous decisions $\hat{\mathbf{V}}_{v}[n],\left(n=\left(N_{\text {wind }}-1\right)(k-1), \cdots,\left(N_{\text {wind }}-1\right) k\right)$. Note that in (10) the factor $\sqrt{1 / 2}$ is included to ensure the validity of (9).

Consequently, both $\mathbf{R}\left[k_{N}\right]$ and $\mathbf{R}_{v}^{i}\left[k_{N}\right]$ may be estimated for the $k$ th length- $N_{\text {wind }}$ block without acquiring the CSI and then tracked recursively in time-varing fading channels as follows:

$$
\begin{aligned}
\mathbf{R}\left[k_{N}\right] & =\beta \mathbf{R}\left[k_{N}-1\right]+(1-\beta) \underline{\mathbf{Y}}^{H}\left[k_{N}\right] \underline{\mathbf{Y}}\left[k_{N}\right], \\
\mathbf{R}_{v}^{i}\left[k_{N}\right] & =\beta \mathbf{R}_{v}^{i}\left[k_{N}-1\right]+(1-\beta) \underline{\mathbf{E}}_{v}^{H}\left[k_{N}\right] \underline{\mathbf{E}}_{v}\left[k_{N}\right],
\end{aligned}
$$

where $0<\beta<1$ is the forgetting factor.

3) Adaptive Implementation of MS-DIS: In practice, rather than carrying out the high-complexity SVD to solve the generalized eigenvalue problem of (8), we apply the modified adaptive Newton algorithm of [8] to recursively update the DIS filter $\mathbf{f}_{v}\left[k_{N}\right]$. This modified adaptive Newton algorithm, which was shown in [8] to have a fast convergence and an excellent tracking capability, may be summarized based on (11) and (12) as follows:

$\mathbf{P}_{v}\left[k_{N}+1\right]=\frac{1}{\beta}\left(\mathbf{P}_{v}\left[k_{N}\right]-\frac{\mathbf{P}_{v}\left[k_{N}\right] \underline{\mathbf{E}}_{v}^{H}\left[k_{N}+1\right] \underline{\mathbf{E}}_{v}\left[k_{N}+1\right] \mathbf{P}_{v}\left[k_{N}\right]}{\operatorname{Trace}\left(\mu \mathbf{I}+\underline{\mathbf{E}}_{v}\left[k_{N}+1\right] \mathbf{P}_{v}\left[k_{N}\right] \underline{\mathbf{E}}_{v}^{H}\left[k_{N}+1\right]\right)}\right.$ $\mathbf{a}_{v}\left[k_{N}+1\right]=\underline{\mathbf{Y}}\left[k_{N}+1\right] \mathbf{f}_{v}\left[k_{N}\right]$,

$\mathbf{r}_{v}\left[k_{N}+1\right]=\beta \mathbf{r}_{v}\left[k_{N}+1\right]+(1-\beta) \underline{\mathbf{Y}}^{H}\left[k_{N}+1\right] \mathbf{a}_{v}\left[k_{N}+1\right]$,

$b_{v}\left[k_{N}+1\right]=\beta b_{v}\left[k_{N}\right]+(1-\beta) \mathbf{c}_{v}^{H}\left[k_{N}+1\right] \mathbf{c}_{v}\left[k_{N}+1\right]$,

$\tilde{\mathbf{f}}_{v}\left[k_{N}+1\right]=\frac{\mathbf{r}_{v}\left[k_{N}+1\right]}{b_{v}\left[k_{N}+1\right]}$,

$\mathbf{f}_{v}\left[k_{N}+1\right]=\frac{2 \mathbf{P}_{v}\left[k_{N}+1\right] \tilde{\mathbf{f}}_{v}\left[k_{N}+1\right]}{1+\tilde{\mathbf{f}}_{v}^{H}\left[k_{N}+1\right] \mathbf{P}_{v}\left[k_{N}+1\right] \tilde{\mathbf{f}}_{v}\left[k_{N}+1\right]}$.

For algorithm initialization, we simply adopt $\mathbf{P}_{v}[1]=0.01 \mathbf{I}_{N_{r}}$, $\mathbf{f}_{v}[1]=\mathbf{r}_{v}[1]=\frac{1}{N_{r}}[11 \cdots 1]^{T}$ and $d[1]=1$. Accordingly, the filter $f_{v}\left[k_{N}\right]$ is updated at the beginning of each $N_{\text {wind }}$-symbol block and it is used unaltered within the $N_{\text {wind }}$-symbol block to suppress the MAI induced by other MSs. In order to assess the MAI suppression capability of the multiple-symbol-based adaptive DIS scheme, we examine the SINR loss (SINRL) at the output of the DIS filter $\mathbf{f}_{v}\left[k_{N}\right]$ in comparison to that achieved by the theoretically optimal filter $\mathbf{f}_{v}^{o}\left[k_{N}\right]$, which is obtained under the assumption of having perfect channel knowledge at the receiver. More specifically, the SINRL for the $k_{N}$ th $N_{\text {wind }}$-symbol block can be expressed in $\mathrm{dB}$ as:

$\mathrm{SINRL} \triangleq 10 \log \left(\frac{\mathbf{f}_{v}^{o H}\left[k_{N}\right] \mathbf{R}\left[k_{N}\right] \mathbf{f}_{v}^{o}\left[k_{N}\right] \mathbf{f}_{v}^{H}\left[k_{N}\right] \mathbf{R}_{v}^{i}\left[k_{N}\right] \mathbf{f}_{v}\left[k_{N}\right]}{\mathbf{f}_{v}^{o H}\left[k_{N}\right] \mathbf{R}_{v}^{i}\left[k_{N}\right] \mathbf{f}_{v}^{o}\left[k_{N}\right] \mathbf{f}_{v}^{H}\left[k_{N}\right] \mathbf{R}\left[k_{N}\right] \mathbf{f}_{v}\left[k_{N}\right]}\right)$

Let us now characterize the SINRL suffered by the multiplesymbol-based adaptive DIS filter in comparison to its single-symbolbased counterpart for both a $2 \times 2$ and for a $8 \times 8$ uncoded DSDMA system in Fig. 2(a). It is observed from Fig. 2(a) that the multiple- 


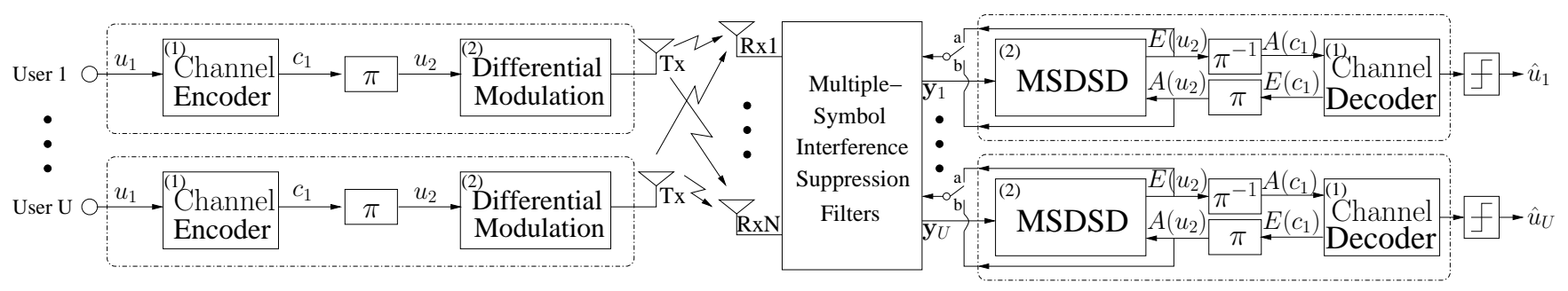

Fig. 1. Multiple-symbol differential spatial division multiple access transceiver architecture.

symbol-based regime may impose a lower filter update complexity at the cost of a slight degradation in its tracking capability. Furthermore, regardless of the value of $N_{\text {wind }}$ employed by the MSINR-based DIS scheme, the conventional RLS-based LMMSE-DIS exhibits a stronger sensitivity to the quality of the feedback decision than that of its MSINR-based counterpart in the decision-directed mode. This results in an inferior tracking capability. On the other hand, as the fading channel becomes increasingly time-selective, in addition to the initial filter training session at the very beginning of transmissions, the training-sequence-aided filter-coefficient refinement process has to be carried out periodically and more frequently, hence imposing an increased filter training overhead. For example, as shown in Fig. 2(b), in the context of an uncoded $2 \times 2$ DSDMA system, $4 \%$ of the transmission resources are occupied by the training session required for maintaining a relatively low SINRL when the normalized Doppler frequency is $f_{d}=0.0001$, while the training symbol overheads increase to $25 \%$ for $f_{d}=0.005$. This is because the filter coefficients rapidly become outdated in severely time-selective fading channels, which results in a rapid symbol error probability (SEP) degradation at the output of the conventional differential detector (CDD), which in turn erodes the MAI suppression capability of the DIS filter in its decision-directed mode.

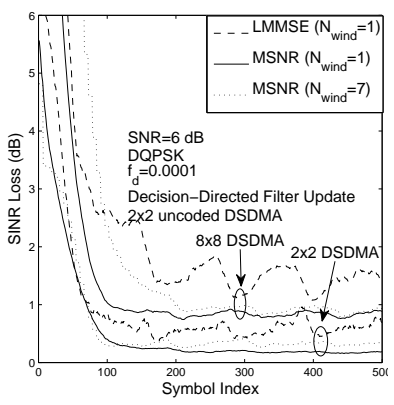

(a) For different $N_{\text {wind }}$.

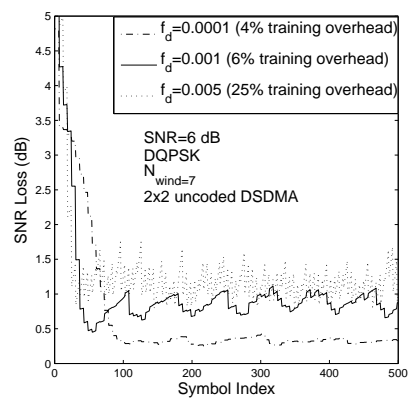

(b) For different $f_{d}$.
Fig. 2. SINRL performance of the multiple-symbol adaptive DIS filter.

\section{B. Multiple-Symbol Differential Sphere Detection}

As mentioned previously, apart from its beneficial complexity reduction, the multiple-symbol-based DIS scheme also facilitates the implementation of the MSDSD detection technique as a benefit of imposing no further distortion to the phase difference between the consecutively transmitted symbols in addition to that caused by the time-varying fading channel. Let us now briefly review the softinput soft-output (SISO) MSDSD scheme, which will be used to generate the soft-bit-information for the desired $v$ th user following the DIS stage. Under the assumption that the interference imposed by all other MSs was significantly mitigated after the DIS processing, the probability density function (PDF) of the DIS filter's output signal $\mathbf{y}_{v}\left[k_{N}\right]$ of (3) was conditioned on the transmitted signal $\mathbf{S}_{v}^{d}\left[k_{N}\right]$, which may be approximated for Rayleigh fading channels as [9] (the $N_{\text {wind }}$-symbol block index $k_{N}$ is omitted here for the sake of notational simplicity): $p\left(\mathbf{y}_{v} \mid \underline{\mathbf{S}}_{v}^{d}\right) \approx \frac{\exp \left(-\mathbf{y}_{v}^{H} \Psi^{-1} \mathbf{y}_{v}\right)}{\operatorname{det}(\pi \Psi)}$, where we have $\Psi=\mathcal{E}\left\{\mathbf{y}_{v} \mathbf{y}_{v}^{H} \mid \underline{\mathbf{S}}_{v}^{d}\right\}=\underline{\mathbf{S}}_{v}^{d} \Sigma_{v} \underline{\mathbf{S}}_{v}^{d H}+2 \sigma_{w}^{2} \mathbf{I}_{T_{b}}$, in which $\Sigma_{v}=\mathcal{E}\left\{\underline{\mathbf{H}}_{v} \underline{\mathbf{H}}_{v}^{H}\right\}$ denotes the $v$ th MS's channel covariance matrix. According to its definition [10], a reduced-complexity computation of the a posteriori Log-Likelihood-Ratio (LLR) associated with the $i$ th transmitted bit $x_{i}$ at the output of the maximum-a-posterori (MAP) based MSDSD [6] may be finally expressed with the aid of Bayes' theorem and the "max-sum" approximation as [11] :

$$
\begin{aligned}
L_{D 2}\left(x_{i}\right)= & \ln \frac{\operatorname{Pr}\left(x_{i}=1 \mid \mathbf{y}_{v}\right)}{\operatorname{Pr}\left(x_{i}=-1 \mid \mathbf{y}_{v}\right)} \\
\approx & -\left\|\mathbf{U} \hat{\mathbf{s}}_{\mathrm{MAP}}^{x_{i}=+1}\right\|^{2}+\ln \left[\operatorname{Pr}\left(\hat{\mathbf{x}}_{\mathrm{MAP}}^{x_{i}=+1}\right)\right] \\
& +\left\|\mathbf{U} \hat{\mathbf{s}}_{\mathrm{MAP}}^{x_{i}=-1}\right\|^{2}-\ln \left[\operatorname{Pr}\left(\hat{\mathbf{x}}_{\mathrm{MAP}}^{x_{i}=-1}\right)\right]
\end{aligned}
$$

where $\mathbf{U}$ is an upper-triangular matrix, which may be obtained as $\mathbf{U} \triangleq\left(\mathbf{F} \operatorname{diag}\left\{\mathbf{y}_{v}\right\}\right)^{*}$, and $\mathbf{F}$ is also an upper-triangular matrix generated using the Cholesky factorization of the matrix $\left(\Sigma_{v}+\right.$ $\left.2 \sigma_{w}^{2} \mathbf{I}_{N_{\text {wind }}}\right)^{-1}$. Thus, thanks to the upper-triangular structure of the matrix $\mathbf{U}$, when evaluating Eq. (15), we may find the multiplesymbol-based vectors $\hat{\mathbf{s}}_{\mathrm{MAP}}^{x_{i}=b}$ and $\hat{\mathbf{x}}_{\mathrm{MAP}}^{x_{i}=b},(b=-1$ or +1$)$, which host the MAP symbol and the corresponding bit estimates, respectively, by the sphere detection (SD) algorithms [11]. Furthermore, $\operatorname{Pr}(\mathbf{x})$ of Eq. (15) is the a priori probability, which may be computed based on the a priori LLRs delivered by the outer channel decoder in an iterative receiver. In the sequel, the extrinsic LLR, $L_{E 2}\left(x_{i}\right)$ may be calculated by excluding the corresponding a priori LLR, $L_{A 2}\left(x_{i}\right)$, from the a posteriori LLR, $L_{D 2}\left(x_{i}\right)$, according to $L_{E 2}\left(x_{i}\right)=$ $L_{D 2}\left(x_{i}\right)-L_{A 2}\left(x_{i}\right)$, which is then exploited by the outer decoder after passing it through the interleaver.

\section{MS-DSDMA TRANSCEIVER DESIGN}

\section{A. Turbo DIS Filter Optimization}

As observed in Fig. 2(b), the decision-direct adaptive DIS scheme may be required to periodically refine the filter-coefficients via the training sequence for the sake of maintaining a desirable performance in time-varying channels. In order to improve the quality of the feedback decision, hence circumventing a potentially excessive training sequence overhead, we propose a channel-code aided turbo DIS scheme for the DSDMA system supporting $U$ MSs, as depicted in Fig. 1. At the transmitter of each MS, a block of $L$ information bits $u_{1}$ is first encoded by the convolutional channel encoder in order to generate the coded bits $c_{1}$, which are interleaved by the interleaver $\pi$. Then the resultant permuted bits $u_{2}$ of Fig. 1 are fed through the bit-to-symbol modulater/mapper. Note that the labels $u$ and $c$ represent the uncoded and coded bits, respectively, corresponding to the specific module indicated by the subscript. At the BS of Fig. 1, which is constituted by three modules, namely the DIS filter bank, the MSDSD and the channel decoder, the extrinsic information may be exchanged amongst the three concantenated components in a number of consecutive iterations. Specifically, as shown in Fig. 1, $A(\cdot)$ represents the a priori information expressed in terms of the LLRs, while $E(\cdot)$ denotes the corresponding extrinsic information. 


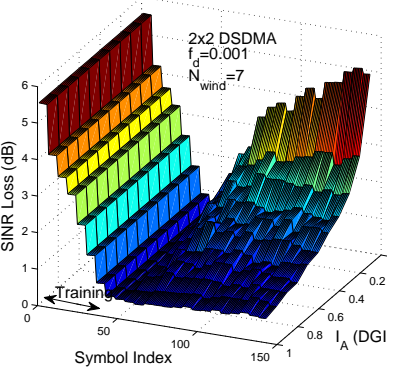

(a) Hard-symbol-decision-direct.

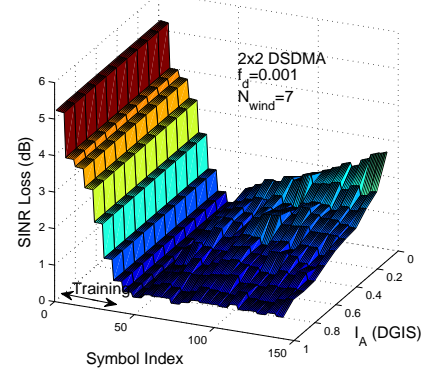

(b) Soft-symbol-decision-direct.
Fig. 3. SINRL performance of the hard-symbol-decision- and soft-symboldecision-direct multiple-symbol DIS filters.

1) Channel-Code-Aided Turbo DIS: At the early stage of the iterative detection process, namely when less confident a priori information is gleaned from the channel decoder in comparison to that provided by the MSDSD (namely when the mutual information (MI) $I_{E\left(u_{1}\right)}$ between the extrinsic value $E\left(u_{1}\right)$ and the bit $u_{1}$ is smaller than that between the extrinsic value $E\left(u_{2}\right)$ and the bit $u_{2}$, i.e. $\left.I_{E\left(u_{2}\right)}\right), \underline{\mathbf{\mathbf { V }}}_{v}\left[k_{N}\right]$ of (10) has to be obtained based on the output of the MSDSD by toggling the decision-directed mode switch to the 'a' location of Fig. 1, if the system is working in the decisiondirected mode. However, as soon as the a priori information becomes more confident during the iterative detection process, namely when we have $I_{E}\left(u_{1}\right)>I_{E}\left(u_{2}\right)$, it is preferred to switch to the "channelcode-aided" decision-directed mode by toggling the switch to the ' $b$ ' location in Fig. 1, so that $\underline{\mathbf{V}}_{v}\left[k_{N}\right]$ of (10) is calculated from the $a$ priori information provided by the channel decoder, for the sake of enhancing the optimization of the DIS filter bank.

2) Soft-Symbol-Decision-Direct DIS: Based on the idea of retaining the valuable soft-information contained in the a posteriori LLRs, which would be simply discarded by the action of subjecting the LLRs to hard decisions, soft-symbol-decision-direct (SSDD) DIS is advocated, where the soft- rather than hard-decision symbol is caculated based on the a priori LLRs delivered either by the MSDSD or by the channel decoder, which in turn is used as our estimate of the transmitted symbol in (10) to adjust the DIS filter's coefficients. In Fig. 3 we visualize the benefits of the SSDD scheme by plotting the filter's SINRL versus both the filter optimization iteration index and the a priori MI measured at its decision feedback branch seen in Fig. 1. Significant SINRL performance gains may be achieved by the SSDD scheme, as observed in Fig. 3, over its hard-symbol-decisiondirected (HSDD) counterpart. More specifically, the effects of the sharply-degraded SINRL experienced by the HSDD-DIS filter when the a priori MI is low may be substantially mitigated by employing the SSDD-based regime. On the other hand, it is also observed from Fig. 3 that the adaptive DIS filter's tracking capability is enhanced for both the DSDD- and SSDD-based techniques, when more confident a priori information becomes available which is expected to become available owing to the beneficial information exchange amongst the concatenated blocks of the three-stage turbo DIS receiver of Fig. 1.

\section{B. Reduced-Complexity MSDSD Design}

In order to reduce the complexity imposed by the MSDSD decoder for the turbo DIS receiver of Fig. 1, two novel complexity reduction techniques, namely the apriori-LLR-threshold (ALT) and adaptivewindow-duration (AWD), are devised in this section by taking advantage of the iterative detection mechanism.

1) Apriori-LLR-Threshold Aided MSDSD: First of all, let us review the definition of the a priori LLRs, which is the logarithm of

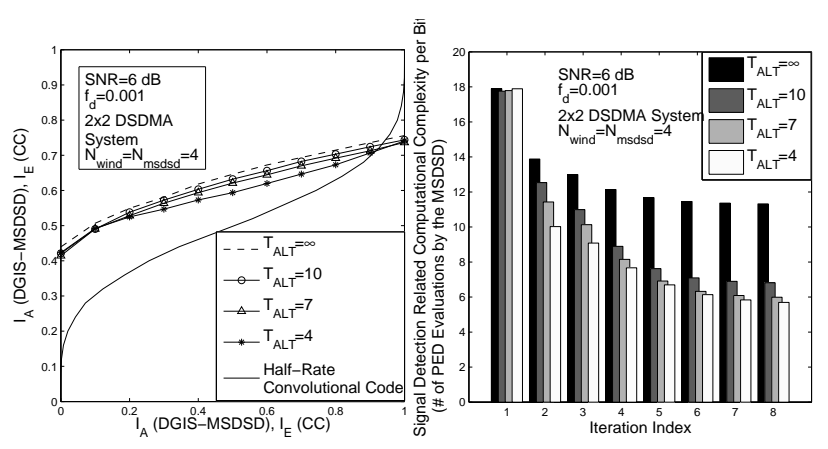

(a) Inner EXIT curves.

(b) Complexity reduction achieved.

Fig. 4. Characterization of the ALT-aided scheme for the MSDSD.

the ratio of the bit probabilities associated with +1 and -1 [10], that may be expressed as $L_{A}\left(x_{j}\right)=\ln \frac{P\left[x_{j}=+1\right]}{P\left[x_{j}=-1\right]}$. Therefore, the sign of the resultant LLRs indicates whether the current bit is more likely to be +1 or -1 , whereas the magnitude reflects how reliable the decision concerning the current bit is. In the light of this, the search space of the MSDSD may be significantly reduced by invoking an ALT controlled technique. To be specific, when calculating the $a$ posteriori LLR $L_{D 2}\left(x_{i}\right)$ of (14) for the $i$ th bit component $x_{i}$ of the bit vector $\mathbf{x}$, the MSDSD search space may be reduced by a factor of $2^{J}$, if the a priori LLRs of $J$ number of bit elements $x_{j}(j \neq i, j \in \mathcal{J})$ delivered by the channel decoder exhibit high magnitudes, which are higher than the preset threshold $T_{\mathrm{ALT}}$, as the iterative detection proceeds. This is because the vector candidates $\mathbf{x}$ associated with $x_{j}(j \in \mathcal{J})$ having values opposite to those indicated by the sign of $L_{A}\left(x_{j}\right)(j \in \mathcal{J})$ are unlikely to be the genuine transmitted bit vector, which may be excluded from the search space.

On the other hand, it is intuitive that the LLR threshold $T_{\mathrm{ALT}}$ cannot be set arbitrarily, since it plays a vital role in determining the system's performance. Thus, the choice of $T_{\mathrm{ALT}}$ is investigated in the context of a $(2 \times 2)$ DSDMA system using $N_{\text {wind }}=4$ at the SNR of $6 \mathrm{~dB}$, where its impacts on the Extrinsic Information Transfer (EXIT) curve [12] of the inner combined "DIS-MSDSD" decoder of Fig. 1 and on the MSDSD's computational complexity are visualized in Figs. 4(a) and 4(b), respectively. It is observed in Fig. 4(b) that although the MSDSD's computational complexity quantified in terms of the number of partial Euclidean distance (PED) evaluations may be reduced by having an increasingly reduced value of $T_{\mathrm{ALT}}$ as the iterations continue, any further complexity reduction achieved is becoming rather marginal when the LLR threshold decreases below $T_{\mathrm{ALT}}=10$. Furthermore, as seen in Fig. 4(a), setting the LLR threshold to a value below $T_{\mathrm{ALT}}=10$ also starts to result in a conspicuously reduced area under the EXIT curve of the amalgamated "DIS-MSDSD" decoder, implying a loss in the system's maximum achievable rate [12]. Hence, based on the observations from Fig. 4, it is desirable to have $T_{\mathrm{ALT}}=10$, which is capable of achieving a significant complexity reduction for the MSDSD without suffering any substantial system performance losses.

2) Adaptive-Window-Duration Based MSDSD: In order to further reduce the complexity imposed by the MSDSD decoder during the iterative decoding process, an AWD based scheme is proposed for the MSDSD, where the observation window size employed by the MSDSD was initially set to the smallest value of $N_{m s d s d}=2$, which will be slightly increased, as soon as the iterative decoding process between the combined "DIS-MSDSD" decoder and the channel decoder converges. The proposed AWD-aided MSDSD scheme is characterized with the aid of the EXIT chart seen in Fig. 5(a), where we may observe the transition of the decision-direct mode from the MSDSD-based mode to the channel-code-based mode at the second 


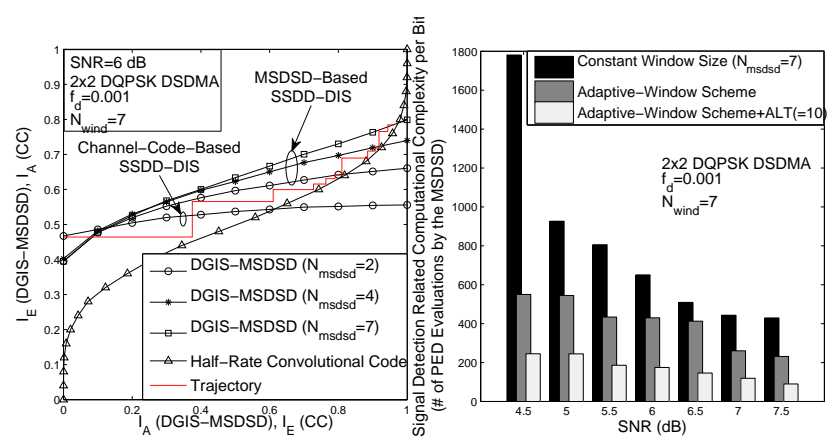

(a) EXIT trajectory.

(b) Complexity reduction achieved

Fig. 5. Characterization of the adaptive-window aided scheme for the MSDSD.

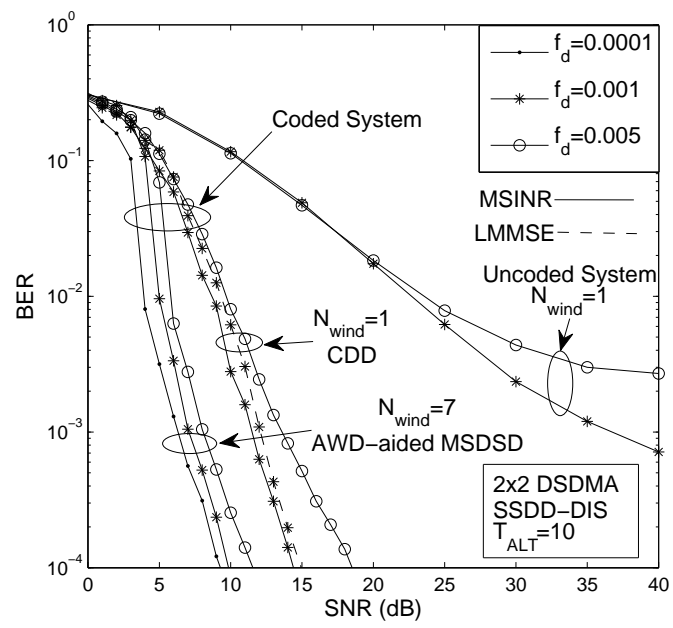

Fig. 6. BER performance of the MS-DSMA system using the ALT- and AWD-aided MSDSD.

iteration, as we discussed in Section IV-A1. Indeed, the complexity imposed by the MSDSD is significantly reduced by the AWD scheme, as observed in Fig. 5(b), where the complexity imposed by the MSDSD in terms of the number of the PED evaluations per bit is plotted versus the SNR for the systems operating both with and without the AWD scheme. Remarkably, the complexity imposed by the MSDSD is substantially reduced in Fig. 5(b) with the aid of the AWD scheme, namely by as much as $66 \%$ at the SNR of $4.5 \mathrm{~dB}$, when the open EXIT tunnel created by having $N_{m s d s d}=7$ is rather narrow. This is not unexpected, since although an increased number of iterations may be needed between the "DIS-MSDSD" decoder and the CC decoder to achieve the same amount of iteration gain, when the AWD scheme is employed, the per-iteration complexity imposed by the MSDSD using a reduced $N_{m s d s d}$ is expected to be exponentially reduced, yielding a potentially reduced overall complexity. More noticeably, the combination of the proposed AWD and ALT schemes allows the MSDSD to achieve an identical iterative gain at $\mathrm{SNR}=4.5 \mathrm{~dB}$, despite imposing a substantially reduced computational complexity, which is only about $\frac{1}{9}$ of that required by the conventional MSDSD scheme, as seen in Fig. 5(b).

\section{Simulation Results and Discussions}

In Fig. 6 the BER performance of the proposed turbo MS-DISaided DSDMA system of Fig. 1 is plotted in comparison to those of its LMMSE-based and MSINR-based single-symbol-DIS-aided counterparts, in the specific context where two single-antenna-aided users are assumed to transmit simutaneously to the two-antenna-aided
BS. Owing to the stronger sensitivity of the RLS-based LMMSE filter to the quality of the feedback decision in the decision-directed mode as implied in Fig. 2(a), it is observed in Fig. 6 that for $N_{\text {wind }}=1$ the coded LMMSE-based DSDMA system is slightly inferior to its MSINR-based counterpart in terms of the BER performance within the SNR range of interest. Furthermore, when the MS-DIS scheme operates in conjunction with $N_{\text {wind }}=7$, the MSINR-based system using the ALT- and AWD-aided MSDSD is capable of achieving an SNR gain of $5 \mathrm{~dB}$ over its LMMSE-based counterpart at the BER target of $10^{-4}$ in the channel-coded scenario associated with $f_{d}=0.001$. Finally, observe in Fig. 6 that the error-floor induced by a more severely time-selective channel may be significantly mitigated by the proposed MSINR-based MS-DIS scheme in conjunction with the ALT- and AWD-aided MSDSD. More specifically, an SNR gain of about $7 \mathrm{~dB}$ can be achieved by the proposed turbo MS-DIS-aided three-stage receiver employing $N_{\text {wind }}=7$ in comparison to the conventional MSINR-based DIS-assisted system using $N_{\text {wind }}=1$ in the time-varying fading channel associated with $f_{d}=0.005$.

\section{CONCLUSION}

In this paper, a turbo MS-DIS-aided three-stage receiver employing the MSDSD was proposed for the DSDMA system, which is suitable for low-complexity and high-bandwidth-efficiency applications in time-varying fading channels, where no channel estimation is required. With the aid of the ALT- and AWD-aided MSDSD scheme devised, the MS-DSDMA system is capable of achieving a significant performance improvements over both its conventional LMMSEand MSINR-based DIS assisted counterparts, while imposing an affordable computational complexity.

\section{REFERENCES}

[1] L. Hanzo, M. Munster, B. J. Choi, and T. Keller, OFDM and MC-CDMA for Broadband Multi-User Communications, WLANs and Broadcasting. IEEE Press, 2003.

[2] T. Weber, A. Sklavos, and M. Meurer, "Imperfect channel-state information in mimo transmission," IEEE Transactions on Communications, vol. 54, pp. 543-552, Mar. 2006.

[3] H. V. Poor and G. W. Wornell, Wireless Communications: Signal Processing Perspectives. Prentice Hall PTR, 1998.

[4] S. K. Cheung and R. Schober, "Differential spatial multiplexing," IEEE Transactions on Wireless Communications, vol. 5, pp. 2127-2135, Aug. 2006.

[5] H.-J. Su and E. Geraniotis, "Maximum signal-to-noise ratio array processing for space-time coded systems," IEEE Transactions on Communications, vol. 50, pp. 1419-1422, Sept. 2002.

[6] L. Lampe, R. Schober, V. Pauli, and C. Windpassinger, "Multiple-symbol differential sphere decoding," IEEE Transactions on Communications, vol. 12, pp. 1981-1985, Dec. 2005.

[7] R. A. Horn and C. R. Johnson, Matrix Analysis. The Press Syndicate of the University of Cambridge, 1999.

[8] J. Yang, F. Yang, H.-S. Xi, W. Guo, and Y. Sheng, "Robust adaptive modified newton algorithm for generalized eigendecomposition and its application," EURASIP Journal on Advances in Signal Processing, vol. 2007, pp. 1-10, June 2007.

[9] R.-R. Chen, R. Koetter, U. Madhow, and D. Agrawal, "Joint noncoherent demodulation and decoding for the block fading channel: a practical framework for approaching shannon capacity," IEEE Transactions on Communications, vol. 51, pp. 1676-1689, Oct. 2003.

[10] L. Hanzo, T. H. Liew, and B. L. Yeap, Turbo Coding, Turbo Equalisation and Space-Time Coding for Transmission over Fading Channels. IEEE Press, 2002.

[11] V. Pauli, L. Lampe, and R. Schober, “"'Turbo DPSK” using soft multiplesymbol differential sphere decoding," IEEE Transactions on Information Theory, vol. 52, no. 4, pp. 1385-1398, 2006.

[12] A. Ashikhmin, G. Kramer, and S. ten Brink, "Extrinsic information transfer functions: model and erasure channel properties," IEEE Transactions on Information Theory, vol. 50, pp. 2657-2673, Nov. 2004. 